\title{
Guest editorial preface: Computational intelligence for neuro-oncological diagnosis
}

\author{
H O R A CIO GONZÁ LEZ-VÉLEZ \\ School of Computing and IDEAS Research Institute, Robert Gordon University, St Andrew Street, Aberdeen AB25 1HG, UK; \\ e-mail: h.gonzalez-velez@rgu.ac.uk
}

\section{Introduction}

The diagnosis of brain tumours is typically based on clinical symptoms, radiological appearance and, often, a histopathological diagnosis of a biopsy. Since treatment of histologically or radiologically similar tumours can vary widely according to their specific nature and patient's characteristics, accurate non-invasive diagnosing techniques are highly sought after in the neuro-oncological practice.

Computational intelligence can arguably contribute to enhancing current imaging and spectroscopybased diagnosis of brain tumours by applying heuristic methods that can learn, adapt, and evolve over time. In particular, this special issue reports the state of the art in intelligent agents, security, knowledge representation, machine learning, clinical data management, and interactive user interfaces in the context of the HealthAgents project.

Funded by the European Commission from January 2006 to December 2008, HealthAgents was a successful trans-European interdisciplinary research project which employed over 50 researchers for the improvement of brain tumour diagnosis (Arús et al., 2006). The HealthAgents consortium evolved from a cross-disciplinary collaboration among European partners, to a unique amalgam of areas of expertise. It comprised two commercial companies, one research institute, and six universities, and concentrated a unique group of well-accomplished, highly computerliterate researchers, most with doctoral degrees in biomedicine, radiology, biology, mathematics, or computer science.

The HealthAgents project created a distributed multi-agent decision support system (DSS) to provide advanced functionalities of machine learning for the analysis and interpretation of brain tumour imaging and spectral data (González-Vélez et al., 2009). The endeavour included a large network of interconnected databases containing the clinical, histological, and molecular phenotype data of brain tumour patients. The DSS facilitated evidence-based clinical decision-making, using magnetic resonance spectroscopy and genetic-based tumour classifications, and included new criteria from the automated analysis of each local database. The HealthAgents project had ensured that all information about patients was kept confidential and stored securely, and provided a clinically validated graphical user interface.

\section{Contents of this issue}

This special issue contains six original articles and intends to reflect the project's progress from the standpoints of the different research groups involved. The main aim of this issue is to illustrate how the HealthAgents project has contributed to advance the field of computational intelligence applied to neuro-oncological diagnosis. Nevertheless, it is important to emphasize that all the distinct research contributions have been organized as coordinated building blocks of the HealthAgents DSS, the raison d'être of the entire project.

Entitled 'A knowledge-rich distributed decision support framework: a case study for brain tumour diagnosis' by Dupplaw et al., the first contribution adumbrates the HealthAgents framework with special emphasis on the intelligent agent and semantic web technologies employed to interconnect the 
contributing clinical centres. The framework underpins all the software development of the DSS, since it deploys the foundations for the data exchange among brain tumour classifiers and repositories on a local and global basis.

The second contribution is 'The design and implementation of a novel security model for HealthAgents' by Xiao et al. and describes the HealthAgents multi-layer security model. It identifies the link-anonymized data transportation, sharing and collection services as well as the access control mechanism. The model had to comply not only with the DSS technical requirements, but also with the legal regulations of the different countries involved.

In the third article of this special issue, 'A generic and extensible automatic classification framework applied to brain tumour diagnosis in HealthAgents', Sáez et al. enumerate the pattern recognition methods employed in the classification of brain tumours within the DSS. Two classification nodes located in Belgium and Spain furnished 25 new classifiers for multiple binary and multi-class clinical questions regarding brain tumour diagnosis, and ranked those classifiers based on their performance and suitability for the clinical case in hand.

Used to represent knowledge across the project, the HealthAgents ontology is explained by $\mathrm{Hu}$ et al. in the fourth manuscript 'The HealthAgents ontology: knowledge representation in a distributed DSS for brain tumours'. The ontology played three crucial roles in the DSS: the common vocabulary for different databases, the shared conceptual repository for clinical terminology, and the translation template for legacy/existing data descriptors.

Described in the fifth article, 'A web accessible distributed data warehouse for brain tumour diagnosis', authored by Estanyol et al., the database allows the DSS to be integrated with the graphical user interface to trigger the different classifiers. This database can be understood as a clinical tool providing clinical history, medical control, and medical experiment management.

Finally, 'The development of a graphical user interface, functional elements and classifiers for the non-invasive characterization of childhood brain tumours using magnetic resonance spectroscopy' by Gibb et al. outlines the design of the interactive clinical user interface of the project, which acted as the DSS front-end. As opposed to previous interfaces employed in medical imaging, the HealthAgents approach entailed an active participation of clinicians and was validated using a sample of children with cerebellar malignancies.

In summary, by providing a distributed agent-based evidence-based DSS, the HealthAgents project has broadened the ambit of machine learning classification, allowing classifiers to be built using geographically dispersed data sources, while keeping patient anonymity and data integrity. The HealthAgents project has deployed an evidence-based heuristic approach for the diagnosis of heterogeneous brain tumour types, displayed through an interactive graphical user interface that fitted the neuro-oncological practice.

\section{Acknowledgements}

The HealthAgents project was initially coordinated by Mariola Mier in 2006 and, subsequently, by Magi Lluch-Ariet. The scientific, clinical, and dissemination management duties were overseen by Paul Lewis, Andrew Peet, and Horacio González-Vélez, respectively. The principal investigators and work package leaders in the project were Carles Arús, Theodoros Arvanitis, Bernardo Celda, Srinandan Dasmahapatra, Juan Miguel García-Gómez, Sabine Van Huffel, Margarida Julià-Sapé, Montserrat Robles, Giulia Valsecchi, and Bonnie Webber.

Special thanks are owed to the following reviewers for their valuable suggestions and comments on the articles in this special issue:

- K. Byrne, University of Edinburgh

- M. Chan, University of Edinburgh

- L. Chen, University of Ulster at Jordanstown

- G. Dick, University of Otago

- X. Franch, Universitat Politècnica de Catalunya

- D. Greer, Queen's University Belfast 
- L. Kurgan, University of Alberta

- S-W. Lee, University of North Carolina at Charlotte

- F. McNeill, University of Edinburgh

- B. Menze, Massachusetts Institute of Technology

- L. Moody, Coventry University

- A. Moreno, Universitat Rovira i Virgili

- M. Schumacher, Haute Ècole Spécialisée de Suisse Occidentale

- E. Sillence, Northumbria University

- F. Thabtah, University of Huddersfield

- T. Veale, University College Dublin

- A. Vellido, Universitat Politècnica de Catalunya

- A. Wright, St. George's University of London

- G. Zhao, Sun Yat-sen University

A special mention to Samantha Lyle of EnglishProofreading.co.uk for her precise attention to detail when proofreading the reviewed papers. The entire HealthAgents project was funded by the Information Society Technologies priority of the European Commission Sixth Framework Programme as a Specific Targeted Research Project with contract number IST-2004-272141 from 1 January 2006 to 31 December 2008.

\section{References}

Arús, C., Celda, B., Dasmahapatra, S., Dupplaw, D., González-Vélez, H., Van Huffel, S., Lewis, P., Lluch i Ariet, M., Mier, M., Peet, A. \& Robles, M. 2006. On the design of a web-based decision support system for brain tumour diagnosis using distributed agents. In 2006 IEEE/WIC/ACM International Conference on Web Intelligence \& Intelligent Agent Technology (WI-IAT 2006 Workshop). Hong Kong 208-211.

González-Vélez, H., Mier, M., Julià-Sapé, M., Arvanitis, T. N., García-Gómez, J. M., Robles, M., Lewis, P. H., Dasmahapatra, S., Dupplaw, D., Peet, A., Arús, C., Celda, B., Van Huffel, S. \& Lluch-Ariet, M. 2009. HealthAgents: distributed multi-agent brain tumor diagnosis and prognosis. Applied Intelligence 30(3), 191-202. 